Article

\title{
Ulam Stability for a Class of Hill's Equations
}

\author{
Ryuma Fukutaka and Masakazu Onitsuka * \\ Department of Applied Mathematics, Okayama University of Science, Okayama 700-0005, Japan; s14m086fr@ous.jp \\ * Correspondence: onitsuka@xmath.ous.ac.jp
}

Received: 28 October 2019; Accepted: 1 December 2019; Published: 5 December 2019

\begin{abstract}
This paper deals with Ulam's type stability for a class of Hill's equations. In the two assertions of the main theorem, we obtain Ulam stability constants that are symmetrical to each other. By combining the obtained results, a necessary and sufficient condition for Ulam stability of a Hill's equation is established. The results are generalized to nonhomogeneous Hill's equations, and then application examples are presented. In particular, it is shown that if the approximate solution is unbounded, then there is an unbounded exact solution.
\end{abstract}

Keywords: Ulam stability; Hill's equation; linear differential equation; periodic coefficient; necessary and sufficient condition

MSC: 34A12; 34A30; 34D10; 39B82

\section{Introduction}

The concept of Ulam's type stability was posed by Ulam, and its development is remarkable. Many researchers have studied this problem for functional equations. For an overview, see the book [1] written by Brzdęk, Popa, Raşa, and Xu. In 1998, Alsina and Ger [2] introduced this concept in the field of differential equations. After that, the study of Ulam's type stability for differential equations continued to grow (see, [3-10]). Recently, Fukutaka and Onitsuka [11,12] dealt with Ulam's type stability of the periodic linear differential equation

$$
y^{\prime}-p(x) y=0
$$

on $\mathbb{R}$, where $p: \mathbb{R} \rightarrow \mathbb{R}$ is a continuous periodic function. Throughout this paper, let $I=(a, b)$, $-\infty \leq a<b \leq \infty$. We say that (1) has "Ulam stability (US)" on $I$ if there exists a constant $K>0$ with the following property: Let $\varepsilon>0$ be a given arbitrary constant, and let $\eta: I \rightarrow \mathbb{R}$ be a continuously differentiable function. If $\left|\eta^{\prime}(x)-p(x) \eta(x)\right| \leq \varepsilon$ holds for all $x \in I$, then there exists a solution $y: \mathbb{R} \rightarrow \mathbb{R}$ of (1) such that $|\eta(x)-y(x)| \leq K \varepsilon$ for all $x \in I$. We call such $K$ a "US constant" for (1) on I. In 2020, Fukutaka and Onitsuka [12] established the following theorem of Ulam's type stability and a necessary and sufficient condition.

Theorem 1. Let $P(x)$ and $Q(x)$ be antiderivatives of $p(x)$ and $e^{-P(x)}$ on $\mathbb{R}$, respectively. Suppose that $p(x)$ is a periodic function with period $\omega>0$ on $\mathbb{R}$. Then the following hold:

(i) if $b=\infty$ and $\int_{0}^{\omega} p(x) d x>0$, then (1) has US with minimum US constant

$$
\max _{x \in(0, \omega]}\left\{\left(\lim _{x \rightarrow \infty} Q(x)\right)-Q(x)\right\} e^{P(x)}
$$


on I;

(ii) if $a=-\infty$ and $\int_{0}^{\omega} p(x) d x<0$, then (1) has US with minimum US constant

$$
\max _{x \in(0, \omega]}\left\{Q(x)-\left(\lim _{x \rightarrow-\infty} Q(x)\right)\right\} e^{P(x)}
$$

on $I$.

Remark 1. Let $a=-\infty$ and $b=\infty$. That is, $I=\mathbb{R}$. Suppose that $p(x)$ is a periodic function with period $\omega>0$ on $\mathbb{R}$. Then it is known that the following facts:

(i) if $\int_{0}^{\omega} p(x) d x>0$, then $\lim _{x \rightarrow \infty} Q(x)<\infty$ exists (see, (Lemma 3.1 [12]));

(ii) if $\int_{0}^{\omega} p(x) d x<0$, then $\lim _{x \rightarrow-\infty} Q(x)>-\infty$ exists (see, (Lemma 3.1 [12]));

(iii) $\left\{\left(\lim _{x \rightarrow \infty} Q(x)\right)-Q(x)\right\} e^{P(x)}$ and $\left\{Q(x)-\left(\lim _{x \rightarrow-\infty} Q(x)\right)\right\} e^{P(x)}$ are periodic functions with period $\omega>0$ (see, (Lemma $2.3[11])$ ).

Theorem 2. Suppose that $p(x)$ is a periodic function with period $\omega>0$ on $\mathbb{R}$. Then (1) has US on $\mathbb{R}$ if and only if $\int_{0}^{\omega} p(x) d x \neq 0$.

In recent years, Ulam stability has been actively studied not only for the first-order linear differential equations but also for the second-order linear differential equations. In 2010, Li [13] dealt with Ulam's type stability of the simple second-order linear differential equation

$$
y^{\prime \prime}=\lambda^{2} y
$$

where $\lambda>0$. This study extends to more general equations with constant coefficient. For example see the works of Li and Huang [14], Li and Shen [15], and Xue [16]. On the other hand, there are many studies on the second-order linear differential equations with variable coefficients (see, [17-24]). It is well known that the most commonly encountered variable coefficient second order differential equation is Hill's equation

$$
y^{\prime \prime}+p(x) y=0
$$

where $p(x)$ is a periodic function. This equation briefly describes the behavior of a large number of physical systems. For example, we can find a pendulum with moving support, electrons in a periodic potential, and beam stabilization in alternating gradient proton synchrotron (see, (Chapter 7 [25])). However, there are no studies on the Ulam stability of the second-order linear differential equations with periodic coefficients. So, this paper focuses on the stability of differential equations with periodic coefficients. The main equation in this paper is

$$
y^{\prime \prime}-\left(\lambda^{2}(x)-\lambda^{\prime}(x)\right) y=0
$$

on $\mathbb{R}$, where $\lambda: \mathbb{R} \rightarrow \mathbb{R}$ is a continuously differentiable, periodic function with period $\omega>0$. Since

$$
\lambda^{\prime}(x+\omega)=\lim _{h \rightarrow 0} \frac{\lambda(x+\omega+h)-\lambda(x+\omega)}{h}=\lim _{h \rightarrow 0} \frac{\lambda(x+h)-\lambda(x)}{h}=\lambda^{\prime}(x)
$$

for all $x \in \mathbb{R}, \lambda^{2}(x)-\lambda^{\prime}(x)$ is also a periodic function with period $\omega>0$ on $\mathbb{R}$, and thus, this equation is a member of Hill's equations. If $\lambda(x) \equiv \lambda$ then the above equation is reduced to the equation $y^{\prime \prime}=\lambda^{2} y$.

The main purpose of this study is to establish a necessary and sufficient condition for Ulam stability of Hill's equations on $\mathbb{R}$. In the second section, we will establish Ulam stability of nonhomogeneous equations. In Section 3, we will give the main theorem and its proof. Also, a instability theorem is given. 
By using the obtained results, a necessary and sufficient condition is established. In Section 4, we will disscus the minimal US constant for the case $\lambda(x) \equiv \lambda$. In addition, a comparison with previous results is also presented. In Section 5, the concept obtained in Section 3 is extended to nonhomogeneous Hill's equations. As an application example, we will discuss Ulam stability of Hill's equation whose coefficient is described by Fourier series. Finally, we will conclude that the unboundedness of the approximate solution implies the unboundedness of the exact solution.

\section{Ulam Stability for Nonhomogeneous Equations}

In this section, we consider the nonhomogeneous first-order linear differential equation

$$
y^{\prime}-p(x) y=f(x)
$$

on $\mathbb{R}$, where $p, f: \mathbb{R} \rightarrow \mathbb{R}$ are continuous. We say that (2) has "Ulam stability (US)" on I if there exists a constant $K>0$ with the following property: Let $\varepsilon>0$ be a given arbitrary constant, and let $\eta: I \rightarrow \mathbb{R}$ be a continuously differentiable function. If $\left|\eta^{\prime}(x)-p(x) \eta(x)-f(x)\right| \leq \varepsilon$ holds for all $x \in I$, then there exists a solution $y: \mathbb{R} \rightarrow \mathbb{R}$ of (2) such that $|\eta(x)-y(x)| \leq K \varepsilon$ for all $x \in I$. We say such $K$ a "US constant" for (2) on $I$. Using the previous result Theorem 1 , we find the following theorem.

Theorem 3. Let $P(x)$ and $Q(x)$ be antiderivatives of $p(x)$ and $e^{-P(x)}$ on $\mathbb{R}$, respectively. Suppose that $p(x)$ is a periodic function with period $\omega>0$ on $\mathbb{R}$. Then the following hold:

(i) if $b=\infty$ and $\int_{0}^{\omega} p(x) d x>0$, then (2) has US with US constant

$$
\max _{x \in(0, \omega]}\left\{\left(\lim _{x \rightarrow \infty} Q(x)\right)-Q(x)\right\} e^{P(x)}
$$

on I;

(ii) if $a=-\infty$ and $\int_{0}^{\omega} p(x) d x<0$, then (2) has US with US constant

$$
\max _{x \in(0, \omega]}\left\{Q(x)-\left(\lim _{x \rightarrow-\infty} Q(x)\right)\right\} e^{P(x)}
$$

on $I$.

Proof. First, we will prove (i). Suppose that $b=\infty$ and $\int_{0}^{\omega} p(x) d x>0$. Let $u(x)$ be a solution of (2) on $\mathbb{R}$. Then we have

$$
\begin{aligned}
\varepsilon & \geq\left|\eta^{\prime}(x)-p(x) \eta(x)-f(x)\right|=\left|\eta^{\prime}(x)-p(x) \eta(x)-\left(u^{\prime}(x)-p(x) u(x)\right)\right| \\
& =\left|(\eta(x)-u(x))^{\prime}-p(x)(\eta(x)-u(x))\right|
\end{aligned}
$$

on $I$. Using Theorem 1 with the above inequality, the periodicity of $p(x)$ and $\int_{0}^{\omega} p(x) d x>0$, we see that there exists a solution $v(x)$ of (1) such that

$$
|(\eta(x)-u(x))-v(x)| \leq \max _{x \in(0, \omega]}\left\{\left(\lim _{x \rightarrow \infty} Q(x)\right)-Q(x)\right\} e^{P(x)} \varepsilon
$$

on $I$. Now, we consider the function $y(x)=u(x)+v(x)$ for all $x \in \mathbb{R}$. Then

$$
y^{\prime}(x)-p(x) y(x)=u^{\prime}(x)-p(x) u(x)+v^{\prime}(x)-p(x) v(x)=f(x) .
$$


This means that $y(x)$ is a solution of (2) such that

$$
|\eta(x)-y(x)|=|(\eta(x)-u(x))-v(x)| \leq \max _{x \in(0, \omega]}\left\{\left(\lim _{x \rightarrow \infty} Q(x)\right)-Q(x)\right\} e^{P(x)} \mathcal{\varepsilon}
$$

on $I$. The proof of (ii) is omitted because it can be proved by the same way.

\section{Main Results}

We consider a Hill's equation of the form

$$
y^{\prime \prime}-\left(\lambda^{2}(x)-\lambda^{\prime}(x)\right) y=0,
$$

on $\mathbb{R}$, where $\lambda: \mathbb{R} \rightarrow \mathbb{R}$ is a continuously differentiable, periodic function with period $\omega>0$. We say that (3) has "Ulam stability (US)" on I if there exists a constant $K>0$ with the following property: Let $\varepsilon>0$ be a given arbitrary constant, and let $\eta: I \rightarrow \mathbb{R}$ be a twice-continuously differentiable function. If $\left|\eta^{\prime \prime}(x)-\left(\lambda^{2}(x)-\lambda^{\prime}(x)\right) \eta(x)\right| \leq \varepsilon$ holds for all $x \in I$, then there exists a solution $y: \mathbb{R} \rightarrow \mathbb{R}$ of (3) such that $|\eta(x)-y(x)| \leq K \varepsilon$ for all $x \in I$. We call such $K$ a "US constant" for (3) on $I$.

When $I=\mathbb{R}$, we can establish the following theorem.

Theorem 4. Let $\Lambda(x), \Gamma^{+}(x)$ and $\Gamma^{-}(x)$ be antiderivatives of $\lambda(x), e^{\Lambda(x)}$ and $e^{-\Lambda(x)}$ on $\mathbb{R}$, respectively. Suppose that $\lambda(x)$ is a periodic function with period $\omega>0$ on $\mathbb{R}$. Then the following hold:

(i) if $\int_{0}^{\omega} \lambda(x) d x>0$, then (3) has US with US constant

$$
\left[\max _{x \in(0, \omega]}\left\{\Gamma^{+}(x)-\left(\lim _{x \rightarrow-\infty} \Gamma^{+}(x)\right)\right\} e^{-\Lambda(x)}\right]\left[\max _{x \in(0, \omega]}\left\{\left(\lim _{x \rightarrow \infty} \Gamma^{-}(x)\right)-\Gamma^{-}(x)\right\} e^{\Lambda(x)}\right]
$$

on $\mathbb{R}$;

(ii) if $\int_{0}^{\omega} \lambda(x) d x<0$, then (3) has US with US constant

$$
\left[\max _{x \in(0, \omega]}\left\{\left(\lim _{x \rightarrow \infty} \Gamma^{+}(x)\right)-\Gamma^{+}(x)\right\} e^{-\Lambda(x)}\right]\left[\max _{x \in(0, \omega]}\left\{\Gamma^{-}(x)-\left(\lim _{x \rightarrow-\infty} \Gamma^{-}(x)\right)\right\} e^{\Lambda(x)}\right]
$$

on $\mathbb{R}$.

Proof. Let $I=\mathbb{R}$. That is, $a=-\infty, b=\infty$. Suppose that twice-continuously differentiable function $\eta(x)$ satisfies $\left|\eta^{\prime \prime}(x)-\left(\lambda^{2}(x)-\lambda^{\prime}(x)\right) \eta(x)\right| \leq \varepsilon$ for all $x \in \mathbb{R}$. Define $\psi(x)=\eta^{\prime}(x)+\lambda(x) \eta(x)$ on $\mathbb{R}$. Since $\lambda(x)$ and $\eta^{\prime}(x)$ are continuously differentiable functions on $\mathbb{R}$, we see that $\psi: \mathbb{R} \rightarrow \mathbb{R}$ is a continuously differentiable function. In addition, we have the inequality

$$
\begin{aligned}
\left|\psi^{\prime}(x)-\lambda(x) \psi(x)\right| & =\left|\left(\eta^{\prime}(x)+\lambda(x) \eta(x)\right)^{\prime}-\lambda(x)\left(\eta^{\prime}(x)+\lambda(x) \eta(x)\right)\right| \\
& =\left|\eta^{\prime \prime}(x)+\lambda(x) \eta^{\prime}(x)+\lambda^{\prime}(x) \eta(x)-\lambda(x)\left(\eta^{\prime}(x)+\lambda(x) \eta(x)\right)\right| \\
& =\left|\eta^{\prime \prime}(x)-\left(\lambda^{2}(x)-\lambda^{\prime}(x)\right) \eta(x)\right| \leq \varepsilon
\end{aligned}
$$

for all $x \in \mathbb{R}$.

First, we will prove (i). From Theorem 1 (i) with $b=\infty$ and $\int_{0}^{\omega} \lambda(x) d x>0$, and $\left|\psi^{\prime}(x)-\lambda(x) \psi(x)\right| \leq$ $\varepsilon$ on $\mathbb{R}$, there exists a solution $w(x)$ of the differential equation

$$
w^{\prime}-\lambda(x) w=0
$$


such that

$$
\left|\eta^{\prime}(x)-(-\lambda(x)) \eta(x)-w(x)\right|=|\psi(x)-w(x)| \leq \max _{x \in(0, \omega]}\left\{\left(\lim _{x \rightarrow \infty} \Gamma^{-}(x)\right)-\Gamma^{-}(x)\right\} e^{\Lambda(x)} \mathcal{\varepsilon}
$$

for all $x \in \mathbb{R}$. From Theorem 3 (ii) with $a=-\infty$ and $\int_{0}^{\omega}(-\lambda(x)) d x<0$, there exists a solution $z(x)$ of the differential equation

$$
z^{\prime}(x)-(-\lambda(x)) z(x)-w(x)=0
$$

such that

$$
|\eta(x)-z(x)| \leq\left[\max _{x \in(0, \omega]}\left\{\Gamma^{+}(x)-\left(\lim _{x \rightarrow-\infty} \Gamma^{+}(x)\right)\right\} e^{-\Lambda(x)}\right]\left[\max _{x \in(0, \omega]}\left\{\left(\lim _{x \rightarrow \infty} \Gamma^{-}(x)\right)-\Gamma^{-}(x)\right\} e^{\Lambda(x)}\right] \varepsilon
$$

for all $x \in \mathbb{R}$. Since $\lambda(x)$ and $w(x)$ are differentiable function, $z^{\prime}(x)$ is also differentiable. Then we have

$$
\begin{aligned}
z^{\prime \prime}(x)-\left(\lambda^{2}(x)-\lambda^{\prime}(x)\right) z(x) & =z^{\prime \prime}(x)+\lambda(x) z^{\prime}(x)+\lambda^{\prime}(x) z(x)-\lambda(x)\left(z^{\prime}(x)+\lambda(x) z(x)\right) \\
& =\left(z^{\prime}(x)+\lambda(x) z(x)\right)^{\prime}-\lambda(x)\left(z^{\prime}(x)+\lambda(x) z(x)\right) \\
& =w^{\prime}(x)-\lambda(x) w(x)=0
\end{aligned}
$$

for all $x \in \mathbb{R}$, and therefore, $z(x)$ is a solution of (3).

Next, we will prove (ii). From Theorem 1 (ii) with $a=-\infty$ and $\int_{0}^{\omega} \lambda(x) d x<0$, and $\mid \psi^{\prime}(x)-$ $\lambda(x) \psi(x) \mid \leq \varepsilon$ on $\mathbb{R}$, there exists a solution $w(x)$ of (4) such that

$$
\left|\eta^{\prime}(x)-(-\lambda(x)) \eta(x)-w(x)\right|=|\psi(x)-w(x)| \leq \max _{x \in(0, \omega]}\left\{\Gamma^{-}(x)-\left(\lim _{x \rightarrow-\infty} \Gamma^{-}(x)\right)\right\} e^{\Lambda(x)} \mathcal{\varepsilon}
$$

for all $x \in \mathbb{R}$. From Theorem 3 (i) with $b=\infty$ and $\int_{0}^{\omega}(-\lambda(x)) d x>0$, there exists a solution $z(x)$ of (5) such that

$$
|\eta(x)-z(x)| \leq\left[\max _{x \in(0, \omega]}\left\{\left(\lim _{x \rightarrow \infty} \Gamma^{+}(x)\right)-\Gamma^{+}(x)\right\} e^{-\Lambda(x)}\right]\left[\max _{x \in(0, \omega]}\left\{\Gamma^{-}(x)-\left(\lim _{x \rightarrow-\infty} \Gamma^{-}(x)\right)\right\} e^{\Lambda(x)}\right] \varepsilon
$$

for all $x \in \mathbb{R}$. By the same calculation as (6), we conclude that $z(x)$ is a solution of (3) on $\mathbb{R}$. This completes the proof of Theorem 4 .

Remark 2. If we compare US constants in (i) and (ii), respectively, it can be seen that they have symmetry. Now we will show this fact below. For $i \in\{1,2\}$, let $\Lambda_{i}(x), \Gamma_{i}^{+}(x)$ and $\Gamma_{i}^{-}(x)$ be antiderivatives of $\lambda_{i}(x), e^{\Lambda_{i}(x)}$ and $e^{-\Lambda_{i}(x)}$ on $\mathbb{R}$, respectively. In (i), If $\lambda(x):=\lambda_{1}(x)$ then we can find the assumption $\int_{0}^{\omega} \lambda_{1}(x) d x>0$ and US constant

$$
K_{1}:=\left[\max _{x \in(0, \omega]}\left\{\Gamma_{1}^{+}(x)-\left(\lim _{x \rightarrow-\infty} \Gamma_{1}^{+}(x)\right)\right\} e^{-\Lambda_{1}(x)}\right]\left[\max _{x \in(0, \omega]}\left\{\left(\lim _{x \rightarrow \infty} \Gamma_{1}^{-}(x)\right)-\Gamma_{1}^{-}(x)\right\} e^{\Lambda_{1}(x)}\right] .
$$

If $\lambda_{1}(x):=-\lambda_{2}(x)$ then this assumption and US constant imply that $\int_{0}^{\omega} \lambda_{2}(x) d x<0$ and

$$
K_{1}=\left[\max _{x \in(0, \omega]}\left\{\Gamma_{2}^{-}(x)-\left(\lim _{x \rightarrow-\infty} \Gamma_{2}^{-}(x)\right)\right\} e^{\Lambda_{2}(x)}\right]\left[\max _{x \in(0, \omega]}\left\{\left(\lim _{x \rightarrow \infty} \Gamma_{2}^{+}(x)\right)-\Gamma_{2}^{+}(x)\right\} e^{-\Lambda_{2}(x)}\right]
$$


since $\Lambda_{1}(x)=-\Lambda_{2}(x)+c_{1}, \Gamma_{1}^{+}(x)=e^{c_{1}} \Gamma_{2}^{-}(x)+c_{2}$ and $\Gamma_{1}^{-}(x)=e^{-c_{1}} \Gamma_{2}^{+}(x)+c_{3}$, where $c_{1}, c_{2}$ and $c_{3}$ are real constants. That is, if $\lambda_{1}(x):=-\lambda_{2}(x)$ then the assumption and US constant in (i) correspond to those in (ii). Therefore, it can be concluded that the assumptions and US constants in (i) and (ii) are symmetric with each other.

Lemma 1. Let $\Lambda(x)$ be an antiderivative of $\lambda(x)$ on $\mathbb{R}$. If $\lambda(x)$ is a periodic function with period $\omega>0$ on $\mathbb{R}$, then

$$
\Lambda(x+\omega)-\Lambda(x)=\int_{0}^{\omega} \lambda(x) d x
$$

holds on $\mathbb{R}$.

Proof. Since $\lambda(x)$ is continuous, and $\Lambda(x)$ is an antiderivative of $\lambda(x)$, there exists a constant $c \in \mathbb{R}$ such that

$$
\Lambda(x)=\int_{0}^{x} \lambda(s) d s+c
$$

for all $x \in \mathbb{R}$. Using this fact, we can prove this lemma. For the reference, see (Lemma 2.1 [12]).

Next, we will present an instability theorem.

Theorem 5. Suppose that $\lambda(x)$ is a periodic function with period $\omega>0$ on $\mathbb{R}$. If $\int_{0}^{\omega} \lambda(x) d x=0$, and $a=-\infty$ or $b=\infty$, then (3) does not have Ulam stability on $I$.

Proof. Set $\Lambda(x)$ be an antiderivative of $\lambda(x)$ on $\mathbb{R}$. Using Lemma 1 and $\int_{0}^{\omega} \lambda(x) d x=0$, we obtain

$$
\Lambda(x+\omega)-\Lambda(x)=0 .
$$

This says that $\Lambda(x)$ is also a periodic function with period $\omega$, and thus, we have

$$
\min _{x \in(0, \omega]} \Lambda(x) \leq \Lambda(x) \leq \max _{x \in(0, \omega]} \Lambda(x)
$$

For any $\varepsilon>0$, we define the function

$$
\eta(x)=\left\{\varepsilon \int_{0}^{x} e^{2 \Lambda(s)} \int_{0}^{s} e^{-\Lambda(u)} d u d s+\left(c_{1} \int_{0}^{x} e^{2 \Lambda(s)} d s+c_{2}\right)\right\} e^{-\Lambda(x)},
$$

where $c_{1}, c_{2} \in \mathbb{R}$. Since this function is a solution of the equation

$$
\eta^{\prime \prime}(x)-\left(\lambda^{2}(x)-\lambda^{\prime}(x)\right) \eta(x)=\varepsilon,
$$

we find $\left|\eta^{\prime \prime}(x)-\left(\lambda^{2}(x)-\lambda^{\prime}(x)\right) \eta(x)\right|=\varepsilon$. Now, we consider the general solution of (3) which given by $y(x)=\left(c_{3} \int_{0}^{x} e^{2 \Lambda(s)} d s+c_{4}\right) e^{-\Lambda(x)}$, where $c_{3}$ and $c_{4}$ are arbitrary constants. Namely, $y(x)$ means all solutions of (3). From this, we obtain 


$$
\begin{aligned}
|\eta(x)-y(x)|= & \mid\left\{\varepsilon \int_{0}^{x} e^{2 \Lambda(s)} \int_{0}^{s} e^{-\Lambda(u)} d u d s+\left(c_{1} \int_{0}^{x} e^{2 \Lambda(s)} d s+c_{2}\right)\right\} e^{-\Lambda(x)} \\
& -\left(c_{3} \int_{0}^{x} e^{2 \Lambda(s)} d s+c_{4}\right) e^{-\Lambda(x)} \mid \\
= & \left|\varepsilon \int_{0}^{x} e^{2 \Lambda(s)} \int_{0}^{s} e^{-\Lambda(u)} d u d s+\left(c_{1}-c_{3}\right) \int_{0}^{x} e^{2 \Lambda(s)} d s+c_{2}-c_{4}\right| e^{-\Lambda(x)} \\
= & \left|\int_{0}^{x}\left(e^{2 \Lambda(s)} \int_{0}^{s} e^{-\Lambda(u)} d u+\frac{c_{1}-c_{3}}{\varepsilon} e^{2 \Lambda(s)}\right) d s+\frac{c_{2}-c_{4}}{\varepsilon}\right| e^{-\Lambda(x)} \mathcal{\varepsilon}
\end{aligned}
$$

for all $x \in \mathbb{R}$.

Now, we consider the case $b=\infty$. By (7), we have

$$
\int_{0}^{x} e^{-\Lambda(s)} d s \geq e^{-\max _{x \in(0, \omega]} \Lambda(x)} x \geq 0 \quad \text { and } \quad e^{2 \max _{x \in(0, \omega]} \Lambda(x)} \geq e^{2 \Lambda(x)} \geq e^{2 \min _{x \in(0, \omega]} \Lambda(x)}>0
$$

for all $x \geq 0$. This implies,

$$
e^{2 \Lambda(x)} \int_{0}^{x} e^{-\Lambda(s)} d s+\frac{c_{1}-c_{3}}{\varepsilon} e^{2 \Lambda(x)} \geq e^{2 \min _{x \in(0, \omega]} \Lambda(x)-\max _{x \in(0, \omega]} \Lambda(x)} x-\frac{\left|c_{1}-c_{3}\right|}{\varepsilon} e^{2 \max _{x \in(0, \omega]} \Lambda(x)}
$$

for all $x \geq 0$. From this, we see that

$$
\begin{aligned}
& \int_{0}^{x}\left(e^{2 \Lambda(s)} \int_{0}^{s} e^{-\Lambda(u)} d u+\frac{c_{1}-c_{3}}{\varepsilon} e^{2 \Lambda(s)}\right) d s+\frac{c_{2}-c_{4}}{\varepsilon} \\
& \quad \geq \frac{e^{2 \min _{x \in(0, \omega]} \Lambda(x)-\max _{x \in(0, \omega]} \Lambda(x)} x^{2}-\frac{\left|c_{1}-c_{3}\right|}{\varepsilon} e^{2 \max _{x \in(0, \omega]} \Lambda(x)} x+\frac{c_{2}-c_{4}}{\varepsilon}}{2}
\end{aligned}
$$

for all $x \geq 0$, and there exists a $x_{1}>\max \{0, a\}$ such that

$$
\frac{e^{\min _{x \in(0, \omega]} \Lambda(x)-2 \max _{x \in(0, \omega]} \Lambda(x)}}{2} x^{2}-\frac{\left|c_{1}-c_{3}\right|}{\varepsilon} e^{2 \max _{x \in(0, \omega]} \Lambda(x)} x+\frac{c_{2}-c_{4}}{\varepsilon}>x
$$

for all $x \geq x_{1}$. Therefore, using (7) and (8), we obtain

$$
|\eta(x)-y(x)|>x e^{-\Lambda(x)} \mathcal{\varepsilon} \geq x e^{-\max _{x \in(0, \omega]} \Lambda(x)} \mathcal{E}
$$

for all $x \geq x_{1}$, so that, $\lim _{x \rightarrow \infty}|\eta(x)-y(x)|=\infty$. Hence, (3) does not have US on $I=(a, \infty)$. The proof of the case $a=-\infty$ is omitted as it can be proved in the same way.

Remark 3. Let $J$ be an open interval satisfying $J \subset I$. Then Ulam stability on I implies the same on J. On the other hand, instability on J implies the same on I.

Corollary 1. Suppose that $\lambda(x)$ is a periodic function with period $\omega>0$ on $\mathbb{R}$. If $\int_{0}^{\omega} \lambda(x) d x=0$, then (3) does not have Ulam stability on $\mathbb{R}$.

Example 1. Consider the case $\lambda(x)=\cos x$ for $x \in I$. If I $=\mathbb{R}$, then (3) does not have US on $\mathbb{R}$ by using Corollary 1.

Theorem 4 and Corollary 1 give the following necessary and sufficient condition. 
Theorem 6. Suppose that $\lambda(x)$ is a periodic function with period $\omega>0$ on $\mathbb{R}$. Then (3) has US on $\mathbb{R}$ if and only if $\int_{0}^{\omega} \lambda(x) d x \neq 0$.

Example 2. Consider the case

$$
\lambda(x)=\alpha+\beta \cos x
$$

on $\mathbb{R}$, where $\alpha, \beta \in \mathbb{R}$. Then (3) has US on $\mathbb{R}$ if and only if $\alpha \neq 0$, by using Theorem 6 .

\section{Minimal US Constant for the Case $\lambda(x) \equiv \lambda$}

Corollary 2. If $\lambda(x) \equiv \lambda \in \mathbb{R} \backslash\{0\}$, then (3) has US with US constant $1 / \lambda^{2}$ on $\mathbb{R}$.

Proof. Let $\Lambda(x)=\lambda x, \Gamma^{+}(x)=e^{\lambda x} / \lambda, \Gamma^{-}(x)=-e^{-\lambda x} / \lambda$ on $\mathbb{R}$. Then we have

$$
\int_{0}^{\omega} \lambda d x=\lambda \omega>0
$$

if $\lambda>0$, and

$$
\int_{0}^{\omega} \lambda d x=\lambda \omega<0
$$

if $\lambda<0$. Thus, we have

$$
\begin{aligned}
\max _{x \in(0, \omega]} & \left.\left\{\Gamma^{+}(x)-\left(\lim _{x \rightarrow-\infty} \Gamma^{+}(x)\right)\right\} e^{-\Lambda(x)}\right]\left[\max _{x \in(0, \omega]}\left\{\left(\lim _{x \rightarrow \infty} \Gamma^{-}(x)\right)-\Gamma^{-}(x)\right\} e^{\Lambda(x)}\right] \\
= & {\left[\max _{x \in(0, \omega]}\left(\frac{e^{\lambda x}}{\lambda}\right) e^{-\lambda x}\right]\left[\max _{x \in(0, \omega]}\left\{-\left(-\frac{e^{-\lambda x}}{\lambda}\right)\right\} e^{\lambda x}\right] } \\
& =\frac{1}{\lambda^{2}}
\end{aligned}
$$

if $\lambda>0$, and

$$
\begin{aligned}
{\left[\max _{x \in(0, \omega]}\left\{\left(\lim _{x \rightarrow \infty} \Gamma^{+}(x)\right)-\Gamma^{+}(x)\right\} e^{-\Lambda(x)}\right]\left[\max _{x \in(0, \omega]}\left\{\Gamma^{-}(x)-\left(\lim _{x \rightarrow-\infty} \Gamma^{-}(x)\right)\right\} e^{\Lambda(x)}\right] } \\
=\left[\max _{x \in(0, \omega]}\left\{-\left(\frac{e^{\lambda x}}{\lambda}\right)\right\} e^{-\lambda x}\right]\left[\max _{x \in(0, \omega]}\left(-\frac{e^{-\lambda x}}{\lambda}\right) e^{\lambda x}\right] \\
=\frac{1}{\lambda^{2}}
\end{aligned}
$$

if $\lambda<0$. From Theorem 4, we conclude that (3) has US with US constant $1 / \lambda^{2}$.

Lemma 2. Let $\lambda(x)$ is a continuously differentiable function on $\mathbb{R}$, and let $b=\infty$. If

$$
0<\sup _{x \in I}\left|\lambda^{2}(x)-\lambda^{\prime}(x)\right|<\infty,
$$

and (3) has US on I, then minimum US constant on I is at least $1 / \sup _{x \in I}\left|\lambda^{2}(x)-\lambda^{\prime}(x)\right|$.

Proof. Let $\varepsilon>0$ be a given arbitrary constant, and let $\eta(x)$ be a solution of the differential equation

$$
\eta^{\prime \prime}(x)-\left(\lambda^{2}(x)-\lambda^{\prime}(x)\right) \eta(x)=\varepsilon
$$


on $I$. Since (3) has US, there exists a constant $K_{0}>0$ such that $|\eta(x)-y(x)| \leq K_{0} \varepsilon$ on $I$. Now, let $y(x)$ be any solution of (3) such that the above inequality holds. We will show that $K_{0} \geq 1 / \sup _{x \in I}\left|\lambda^{2}(x)-\lambda^{\prime}(x)\right|$. By way of contradiction, we assume that $K_{0}<1 / \sup _{x \in I}\left|\lambda^{2}(x)-\lambda^{\prime}(x)\right|$. From this assumption, we have

$$
\begin{aligned}
\eta^{\prime \prime}(x)-y^{\prime \prime}(x) & =\left(\lambda^{2}(x)-\lambda^{\prime}(x)\right)(\eta(x)-y(x))+\varepsilon \\
& \geq-\left|\left(\lambda^{2}(x)-\lambda^{\prime}(x)\right)(\eta(x)-y(x))\right|+\varepsilon \\
& \geq-\left|\lambda^{2}(x)-\lambda^{\prime}(x)\right| K_{0} \varepsilon+\varepsilon \\
& \geq-\left\{\sup _{x \in I}\left|\lambda^{2}(x)-\lambda^{\prime}(x)\right|\right\} K_{0} \varepsilon+\varepsilon \\
& =\left[\frac{1}{\left\{\sup _{x \in I}\left|\lambda^{2}(x)-\lambda^{\prime}(x)\right|\right\}}-K_{0}\right]\left\{\sup _{x \in I}\left|\lambda^{2}(x)-\lambda^{\prime}(x)\right|\right\} \varepsilon
\end{aligned}
$$

on $I$. Set

$$
M=\left[\frac{1}{\left\{\sup _{x \in I}\left|\lambda^{2}(x)-\lambda^{\prime}(x)\right|\right\}}-K_{0}\right]\left\{\sup _{x \in I}\left|\lambda^{2}(x)-\lambda^{\prime}(x)\right|\right\} \varepsilon>0,
$$

and set $x_{0} \in I$. Then we obtain

$$
\eta^{\prime}(x)-y^{\prime}(x) \geq \int_{x_{0}}^{x} M d s+\eta^{\prime}\left(x_{0}\right)-y^{\prime}\left(x_{0}\right)=M x-M x_{0}+\eta^{\prime}\left(x_{0}\right)-y^{\prime}\left(x_{0}\right)
$$

for all $x>x_{0}$. From this inequality, we have

$$
\begin{aligned}
\eta(x)-y(x) \geq & \int_{x_{0}}^{x}\left(M s-M x_{0}+\eta^{\prime}\left(x_{0}\right)-y^{\prime}\left(x_{0}\right)\right) d s+\eta\left(x_{0}\right)-y\left(x_{0}\right) \\
= & \frac{1}{2} M x^{2}-\left(M x_{0}-\eta^{\prime}\left(x_{0}\right)+y^{\prime}\left(x_{0}\right)\right) x \\
& -\frac{1}{2} M x_{0}^{2}+\left(M x_{0}-\eta^{\prime}\left(x_{0}\right)+y^{\prime}\left(x_{0}\right)\right) x_{0}+\eta\left(x_{0}\right)-y\left(x_{0}\right)
\end{aligned}
$$

for all $x>x_{0}$. Using this inequality, we see that $\lim _{x \rightarrow \infty}|\eta(x)-y(x)|=\infty$. This contradicts the inequality $|\eta(x)-y(x)| \leq K_{0} \varepsilon$ on $I$.

Theorem 7. If $\lambda(x)=\lambda \in \mathbb{R} \backslash\{0\}$ on $\mathbb{R}$, then (3) has Ulam stability with minimum US constant $1 / \lambda^{2}$ on $\mathbb{R}$.

Proof. Let $\lambda(x)=\lambda \in \mathbb{R} \backslash\{0\}$ on $\mathbb{R}$. Using Corollary 2, (3) has US and $1 / \lambda^{2}$ is a US constant on $\mathbb{R}$. From Lemma 2 with $\sup _{x \in \mathbb{R}}\left|\lambda^{2}(x)-\lambda^{\prime}(x)\right|=\lambda^{2}$, we see that the minimum US constant for (3) on $\mathbb{R}$ is at least $1 / \lambda^{2}$. This completes the proof of Theorem 7 .

Remark 4. The decisive difference between the previous study described in the introduction and this study is whether the interval is finite or not. In the previous studies [13-16], they assume that $-\infty<a<b<\infty$. If $I=(a, b)=(-\infty, \infty)=\mathbb{R}$, then Ulam stability cannot be judged by their results because the US constant in the proof of previous theorems depends on $a$ or $b$, and, goes to $\infty$ when $a \rightarrow-\infty$ or $b \rightarrow \infty$. However, our study established Ulam stability on an infinite interval, and obtained the minimum US constant. 


\section{Nonhomogeneous Hill's Equations and Applications}

Consider the nonhomogeneous Hill's equation

$$
y^{\prime \prime}-\left(\lambda^{2}(x)-\lambda^{\prime}(x)\right) y=f(x)
$$

where $f: \mathbb{R} \rightarrow \mathbb{R}$ is continuous. Equation (9) has "Ulam stability (US)" on $I$ if and only if there exists $K>0$ such that the following holds: Let $\varepsilon>0$ be given, and let $\eta: I \rightarrow \mathbb{R}$ be a twice-continuously differentiable function. If $\left|\eta^{\prime \prime}(x)-\left(\lambda^{2}(x)-\lambda^{\prime}(x)\right) \eta(x)-f(x)\right| \leq \varepsilon$ on $I$, then there exists a solution $y(x)$ of (9) such that $|\eta(x)-y(x)| \leq K \varepsilon$ on $I$. $K$ is called a "US constant" for (9) on I. The following result is obtained by using Theorem 4 .

Theorem 8. Let $\Lambda(x), \Gamma^{+}(x)$ and $\Gamma^{-}(x)$ be antiderivatives of $\lambda(x), e^{\Lambda(x)}$ and $e^{-\Lambda(x)}$ on $\mathbb{R}$, respectively. Suppose that $\lambda(x)$ is a periodic function with period $\omega>0$ on $\mathbb{R}$. Then the following hold:

(i) if $\int_{0}^{\omega} \lambda(x) d x>0$, then (9) has US with US constant

$$
\left[\max _{x \in(0, \omega]}\left\{\Gamma^{+}(x)-\left(\lim _{x \rightarrow-\infty} \Gamma^{+}(x)\right)\right\} e^{-\Lambda(x)}\right]\left[\max _{x \in(0, \omega]}\left\{\left(\lim _{x \rightarrow \infty} \Gamma^{-}(x)\right)-\Gamma^{-}(x)\right\} e^{\Lambda(x)}\right]
$$

on $\mathbb{R}$;

(ii) if $\int_{0}^{\omega} \lambda(x) d x<0$, then (9) has US with US constant

$$
\left[\max _{x \in(0, \omega]}\left\{\left(\lim _{x \rightarrow \infty} \Gamma^{+}(x)\right)-\Gamma^{+}(x)\right\} e^{-\Lambda(x)}\right]\left[\max _{x \in(0, \omega]}\left\{\Gamma^{-}(x)-\left(\lim _{x \rightarrow-\infty} \Gamma^{-}(x)\right)\right\} e^{\Lambda(x)}\right]
$$

on $\mathbb{R}$.

Proof. First, we will prove (i). Let $u(x)$ be a solution of (9) on $\mathbb{R}$. Suppose that $\int_{0}^{\omega} \lambda(x) d x>0$ and $\left|\eta^{\prime \prime}(x)-\left(\lambda^{2}(x)-\lambda^{\prime}(x)\right) \eta(x)-f(x)\right| \leq \varepsilon$ on $I$. Then we have

$$
\begin{aligned}
\varepsilon & \geq\left|\eta^{\prime \prime}(x)-\left(\lambda^{2}(x)-\lambda^{\prime}(x)\right) \eta(x)-f(x)\right| \\
& =\left|\eta^{\prime \prime}(x)-\left(\lambda^{2}(x)-\lambda^{\prime}(x)\right) \eta(x)-\left[u^{\prime \prime}(x)-\left(\lambda^{2}(x)-\lambda^{\prime}(x)\right) u(x)\right]\right| \\
& =\left|(\eta(x)-u(x))^{\prime \prime}-\left(\lambda^{2}(x)-\lambda^{\prime}(x)\right)(\eta(x)-u(x))\right|
\end{aligned}
$$

on I. Using Theorem 4 with the above inequality, we see that there exists a solution $v(x)$ of (3) such that

$$
|(\eta(x)-u(x))-v(x)| \leq K_{2} \varepsilon
$$

on $I$, where

$$
K_{2}:=\left[\max _{x \in(0, \omega]}\left\{\Gamma^{+}(x)-\left(\lim _{x \rightarrow-\infty} \Gamma^{+}(x)\right)\right\} e^{-\Lambda(x)}\right]\left[\max _{x \in(0, \omega]}\left\{\left(\lim _{x \rightarrow \infty} \Gamma^{-}(x)\right)-\Gamma^{-}(x)\right\} e^{\Lambda(x)}\right] .
$$

Let $y(x)=u(x)+v(x)$ for all $x \in \mathbb{R}$. Then

$$
y^{\prime \prime}(x)-\left(\lambda^{2}(x)-\lambda^{\prime}(x)\right) y(x)=u^{\prime \prime}(x)-\left(\lambda^{2}(x)-\lambda^{\prime}(x)\right) u(x)+v^{\prime \prime}(x)-\left(\lambda^{2}(x)-\lambda^{\prime}(x)\right) v(x)=f(x) .
$$


Thus, $y(x)$ is a solution of (9) such that

$$
|\eta(x)-y(x)|=|(\eta(x)-u(x))-v(x)| \leq K_{2} \varepsilon
$$

on $I$. The proof of (ii) is omitted because it can be proved by the same way.

Theorem 8 and Corollary 1 imply the following result.

Theorem 9. Suppose that $\lambda(x)$ is a periodic function with period $\omega>0$ on $\mathbb{R}$. Then (9) has US on $\mathbb{R}$ if and only if $\int_{0}^{\omega} \lambda(x) d x \neq 0$.

US constants in Theorems 4 and 8 can be estimated as follows.

Lemma 3. Let $\Lambda(x), \Gamma^{+}(x)$ and $\Gamma^{-}(x)$ be antiderivatives of $\lambda(x), e^{\Lambda(x)}$ and $e^{-\Lambda(x)}$ on $\mathbb{R}$, respectively. Suppose that $\lambda(x)$ is a periodic function with period $\omega>0$ on $\mathbb{R}$. Then the following hold:

(i) if $\lambda(x)>0$ on $\mathbb{R}$, then

$$
\begin{aligned}
\frac{1}{\left(\max _{x \in(0, \omega]} \lambda(x)\right)^{2}} & \leq\left[\max _{x \in(0, \omega]}\left\{\Gamma^{+}(x)-\left(\lim _{x \rightarrow-\infty} \Gamma^{+}(x)\right)\right\} e^{-\Lambda(x)}\right]\left[\max _{t \in(0, \omega]}\left\{\left(\lim _{x \rightarrow \infty} \Gamma^{-}(x)\right)-\Gamma^{-}(x)\right\} e^{\Lambda(x)}\right] \\
& \leq \frac{1}{\left(\min _{x \in(0, \omega]} \lambda(x)\right)^{2}}
\end{aligned}
$$

(ii) if $\lambda(x)<0$ on $\mathbb{R}$, then

$$
\begin{aligned}
\frac{1}{\left(\min _{x \in(0, \omega]} \lambda(x)\right)^{2}} & \leq\left[\max _{x \in(0, \omega]}\left\{\left(\lim _{x \rightarrow \infty} \Gamma^{+}(x)\right)-\Gamma^{+}(x)\right\} e^{-\Lambda(x)}\right]\left[\max _{x \in(0, \omega]}\left\{\Gamma^{-}(x)-\left(\lim _{x \rightarrow-\infty} \Gamma^{-}(x)\right)\right\} e^{\Lambda(x)}\right] \\
& \leq \frac{1}{\left(\max _{x \in(0, \omega]} \lambda(x)\right)^{2}} .
\end{aligned}
$$

Proof. We will prove (i). For simplicity, let

$$
g(x):=\left\{\Gamma^{+}(x)-\left(\lim _{x \rightarrow-\infty} \Gamma^{+}(x)\right)\right\} e^{-\Lambda(x)}
$$

and

$$
h(x):=\left\{\left(\lim _{x \rightarrow \infty} \Gamma^{-}(x)\right)-\Gamma^{-}(x)\right\} e^{\Lambda(x)} .
$$

Then we have

$$
g^{\prime}(x)=\left\{\Gamma^{+}(x)-\left(\lim _{x \rightarrow-\infty} \Gamma^{+}(x)\right)\right\}^{\prime} e^{-\Lambda(x)}-\lambda(x) g(x)=1-\lambda(x) g(x)
$$

and

$$
h^{\prime}(x)=\left\{\left(\lim _{x \rightarrow \infty} \Gamma^{-}(x)\right)-\Gamma^{-}(x)\right\}^{\prime} e^{\Lambda(x)}+\lambda(x) h(x)=-1+\lambda(x) h(x) .
$$

From Remark $1, g(x)$ and $h(x)$ are periodic functions with period $\omega>0$, so that we can find $x_{1} \in(0, \omega]$ such that $g\left(x_{1}\right)=\max _{x \in(0, \omega]} g(x)$. Since $g\left(x_{1}\right)$ is a maximum value of $g(x)$ on $(0, \omega]$, we see that 


$$
0=g^{\prime}\left(x_{1}\right)=1-\lambda\left(x_{1}\right) g\left(x_{1}\right)=1-\lambda\left(x_{1}\right) \max _{x \in(0, \omega]} g(x) .
$$

That is, $\max _{x \in(0, \omega]} g(x)=1 / \lambda\left(x_{1}\right)$. From this and $\lambda(x)>0$ is a periodic function with period $\omega>0$ on $\mathbb{R}$, we have

$$
0<\frac{1}{\max _{x \in(0, \omega]} \lambda(x)} \leq \max _{x \in(0, \omega]} g(x)=\frac{1}{\lambda\left(x_{1}\right)} \leq \frac{1}{\min _{x \in(0, \omega]} \lambda(x)}
$$

on $(0, \omega]$. Let $x_{2} \in \mathbb{R}$ satisfy $h\left(x_{2}\right)=\max _{x \in(0, \omega]} h(x)$, then we have

$$
0=h^{\prime}\left(x_{2}\right)=-1+\lambda\left(x_{2}\right) h\left(x_{2}\right)=-1+\lambda\left(x_{2}\right) \max _{x \in(0, \omega]} h(x) .
$$

That is, $\max _{x \in(0, \omega]} h(x)=1 / \lambda\left(x_{2}\right)$, and so that, we find

$$
0<\frac{1}{\max _{x \in(0, \omega]} \lambda(x)} \leq \max _{x \in(0, \omega]} h(x)=\frac{1}{\lambda\left(x_{2}\right)} \leq \frac{1}{\min _{x \in(0, \omega]} \lambda(x)}
$$

on $(0, \omega]$. Using (10) and (11), we obtain

$$
\frac{1}{\left(\max _{x \in(0, \omega]} \lambda(x)\right)^{2}} \leq\left(\max _{x \in(0, \omega]} g(x)\right)\left(\max _{x \in(0, \omega]} h(x)\right) \leq \frac{1}{\left(\min _{x \in(0, \omega]} \lambda(x)\right)^{2}}
$$

The proof of (ii) is omitted because it can be proved by the same way.

Theorem 8 and Lemma 3 imply the following result.

Theorem 10. Suppose that $\lambda(x)$ is a periodic function with period $\omega>0$ on $\mathbb{R}$. Then the following hold:

(i) if $\lambda(x)>0$ on $\mathbb{R}$, then (9) has US with US constant $1 /\left(\min _{x \in(0, \omega]} \lambda(x)\right)^{2}$;

(ii) if $\lambda(x)<0$ on $\mathbb{R}$, then (9) has US with US constant $1 /\left(\max _{x \in(0, \omega]} \lambda(x)\right)^{2}$.

In many application fields, the coefficient $p(x)$ of the Hill's equation $y^{\prime \prime}+p(x) y=0$ is treated as a periodic function with period $\pi$. It is well known that $p(x)$ can be rewritten by the Fourier series of $p(x)$. That is, Hill's equation is given by

$$
y^{\prime \prime}+\left[\frac{a_{0}}{2}+\sum_{n=1}^{\infty}\left(a_{n} \cos 2 n x+b_{n} \sin 2 n x\right)\right] y=0
$$

where $a_{0}, a_{n}$ and $b_{n}$ are Fourier coefficients. Now, we consider the case

$$
\lambda(x)=\alpha+\beta \cos 2 x+\gamma \sin 2 x,
$$

in (3), where $\alpha, \beta$ and $\gamma$ are real-valued constants. Then we have 


$$
\begin{aligned}
\lambda^{2}(x)-\lambda^{\prime}(x)= & (\alpha+\beta \cos 2 x+\gamma \sin 2 x)^{2}-(-2 \beta \sin 2 x+2 \gamma \cos 2 x) \\
= & \alpha^{2}+2(\alpha \beta-\gamma) \cos 2 x+2(\alpha \gamma+\beta) \sin 2 x+(\beta \cos 2 x+\gamma \sin 2 x)^{2} \\
= & \alpha^{2}+2(\alpha \beta-\gamma) \cos 2 x+2(\alpha \gamma+\beta) \sin 2 x+\beta^{2} \frac{1+\cos 4 x}{2}+\beta \gamma \sin 4 x \\
& +\gamma^{2}-\gamma^{2} \frac{1+\cos 4 x}{2} \\
= & \frac{2 \alpha^{2}+\beta^{2}+\gamma^{2}}{2}+2(\alpha \beta-\gamma) \cos 2 x+\frac{\beta^{2}-\gamma^{2}}{2} \cos 4 x+2(\alpha \gamma+\beta) \sin 2 x+\beta \gamma \sin 4 x .
\end{aligned}
$$

This implies that this case corresponds to the case:

$$
\begin{gathered}
a_{0}=-\left(2 \alpha^{2}+\beta^{2}+\gamma^{2}\right), \quad a_{1}=-2(\alpha \beta-\gamma), a_{2}=-\frac{\beta^{2}-\gamma^{2}}{2}, a_{n}=0(i=3,4,5, \cdots), \\
b_{1}=-2(\alpha \gamma+\beta), \quad b_{2}=-\beta \gamma \quad \text { and } \quad b_{n}=0(i=3,4,5, \cdots) .
\end{gathered}
$$

Let $\eta(x)$ be a solution of (12). Then it is an approximate solution of the nonhomogeneous Hill's equation

$$
y^{\prime \prime}+\left[\frac{a_{0}}{2}+\sum_{n=1}^{\infty}\left(a_{n} \cos 2 n x+b_{n} \sin 2 n x\right)\right] y=f(x), \quad|f(x)| \leq \varepsilon
$$

where $a_{0}, a_{n}$ and $b_{n}$ are the same as above, and $\varepsilon>0$ is given. In fact,

$$
\left|\eta^{\prime \prime}(x)+\left[\frac{a_{0}}{2}+\sum_{n=1}^{\infty}\left(a_{n} \cos 2 n x+b_{n} \sin 2 n x\right)\right] \eta(x)-f(x)\right|=|0-f(x)| \leq \varepsilon
$$

holds on $\mathbb{R}$. Since

$$
\int_{0}^{\pi} \lambda(x) d x=\alpha \pi
$$

holds, (13) has US on $\mathbb{R}$ if and only if $\alpha \neq 0$ by Theorem 9 .

Next we consider the more simple case: $\alpha=-2, \beta=\gamma=1$. Then we have

$$
\lambda(x)=-2+\cos 2 x+\sin 2 x \text { and } \lambda^{2}(x)-\lambda^{\prime}(x)=5-6 \cos 2 t-2 \sin 2 t+\sin 4 t
$$

and $\lambda(x)<0$ for all $x \in \mathbb{R}$. We will show that if the approximate solution is unbounded, then there is an unbounded exact solution. Using Theorem 10, we see that there exists a solution $y(x)$ of (13) satisfying $|\eta(x)-y(x)| \leq K_{3} \varepsilon$ on $\mathbb{R}$, where

$$
K_{3}:=\frac{1}{\left(\max _{x \in(0, \omega]} \lambda(x)\right)^{2}}=\frac{1}{(-2+\sqrt{2})^{2}} .
$$

Since

$$
\eta_{1}(t)=e^{-\Lambda(x)}=\exp \left(2 x-\frac{1}{2} \sin 2 x+\frac{1}{2} \cos 2 x\right)
$$

is an unbounded solution of (12), we conclude that (13) has at least one unbounded solution. 


\section{Conclusions}

In this paper, we have discussed the Ulam stability of periodic linear differential equations of the first order and second order, respectively. First, we have established a sufficient condition for Ulam stability of nonhomogeneous first order equations. Next, by using this result, Ulam stability of a class of Hill's equations is established. In particular, the exact US constant is given in the theorem. If the coefficient is a constant, it turns out to be the minimum US constant. On the other hand, the instability theorem is also given. By combining the obtained results, we have established a necessary and sufficient condition for Ulam stability of a class of Hill's equations. Using the idea of the results obtained first, this theory is extended to nonhomogeneous Hill's equations. Finally, we have discussed the Ulam stability of Hill's equations where the coefficient is described by a Fourier series. By using the obtained results, it can be concluded that the unboundedness of the approximate solution affects the unboundedness of the exact solution.

Author Contributions: These authors contributed equally to this work; conceptualization, R.F. and M.O.; methodology, R.F. and M.O.; investigation, R.F. and M.O.; resources, M.O.; writing-original draft preparation, R.F. and M.O.; writing-review and editing, R.F. and M.O.; supervision, R.F.; project administration, M.O.; funding acquisition, M.O..

Funding: The second author was supported by JSPS KAKENHI Grant Number JP17K14226.

Acknowledgments: These authors would like to thank the referee for carefully reading our manuscript and for giving useful comments.

Conflicts of Interest: The authors declare no conflict of interest.

\section{References}

1. Brzdęk, J.; Popa, D.; Raşa, I.; Xu, B. Ulam stability of Operators. Mathematical Analysis and Its Applications; Academic Press: Cambridge, MA, USA, 2018.

2. Alsina, C.; Ger, R. On some inequalities and stability results related to the exponential function. J. Inequal. Appl. 1998, 2, 373-380. [CrossRef]

3. Onitsuka, M. Hyers-Ulam stability of first order linear differential equations of Carathéodory type and its application. Appl. Math. Lett. 2019, 90,61-68. [CrossRef]

4. Onitsuka, M.; Shoji, T. Hyers-Ulam stability of first-order homogeneous linear differential equations with a real-valued coefficient. Appl. Math. Lett. 2017, 63, 102-108. [CrossRef]

5. Popa, D.; Raşa, I. On the Hyers-Ulam stability of the linear differential equation. J. Math. Anal. Appl. 2011, 381, 530-537. [CrossRef]

6. Popa, D.; Raşa, I. Hyers-Ulam stability of the linear differential operator with nonconstant coefficients. Appl. Math. Comput. 2012, 219, 1562-1568. [CrossRef]

7. Rezaei, H.; Jung, S.-M.; Rassias, T.M. Laplace transform and Hyers-Ulam stability of linear differential equations. J. Math. Anal. Appl. 2013, 403, 244-251. [CrossRef]

8. Wang, G.; Zhou, M.; Sun, L. Hyers-Ulam stability of linear differential equations of first order. Appl. Math. Lett. 2008, 21, 1024-1028. [CrossRef]

9. Zada, B. Uniform exponential stability in the sense of Hyers and Ulam for periodic time varying linear systems. Differ. Equ. Appl. 2018, 10, 227-234. [CrossRef]

10. Zada, A.; Wang, P.; Lassoued, D.; Li, T. Connections between Hyers-Ulam stability and uniform exponential stability of 2-periodic linear nonautonomous systems. Adv. Differ. Equ. 2017, 192, 7. [CrossRef]

11. Fukutaka, R.; Onitsuka, M. Best constant in Hyers-Ulam stability of first-order homogeneous linear differential equations with a periodic coefficient. J. Math. Anal. Appl. 2019, 473, 1432-1446. [CrossRef]

12. Fukutaka, R.; Onitsuka, M. A necessary and sufficient condition for Hyers-Ulam stability of first-order periodic linear differential equations. Appl. Math. Lett. 2020, 100, 106040. [CrossRef]

13. Li, Y. Hyers-Ulam stability of linear differential equations $y^{\prime \prime}=\lambda^{2} y$. Thai J. Math. 2010, 8, 215-219. 
14. Li, Y.; Huang, J. Hyers-Ulam stability of linear second-order differential equations in complex Banach spaces. Electron. J. Differ. Equ. 2013, 184, 7.

15. Li, Y.; Shen, Y. Hyers-Ulam stability of linear differential equations of second order. Appl. Math. Lett. 2010, 23, 306-309. [CrossRef]

16. Xue, J. Hyers-Ulam stability of linear differential equations of second order with constant coefficient. Ital. J. Pure Appl. Math. 2014, 32, 419-424.

17. Abdollahpour, M.R.; Park, C. Hyers-Ulam stability of a class of differential equations of second order. J. Comput. Anal. Appl. 2015, 18, 899-903.

18. Alqifiary, Q.H.; Jung, S.-M. On the Hyers-Ulam stability of differential equations of second order. Abstr. Appl. Anal. 2014, 2014, 483707. [CrossRef]

19. Anderson, D.R. Hyers-Ulam stability of higher-order Cauchy-Euler dynamic equations on time scales. Dyn. Syst. Appl. 2014, 23, 653-664.

20. Ghaemi, M.B.; Gordji, M.E.; Madjid, E.; Alizadeh, B.; Park, C. Hyers-Ulam stability of exact second-order linear differential equations. Adv. Differ. Equ. 2012, 2012, 36. [CrossRef]

21. Javadian, A.; Sorouri, E.; Kim, G.H.; Gordji, M.E. Generalized Hyers-Ulam stability of the second-order linear differential equations. J. Appl. Math. 2011, 2011, 813137. [CrossRef]

22. Shen, Y. An integrating factor approach to the Hyers-Ulam stability of a class of exact differential equations of second order. J. Nonlinear Sci. Appl. 2016, 9, 2520-2526. [CrossRef]

23. Shen, Y.; Chen, W.; Lan, Y. On the Ulam stability of a class of Banach space valued linear differential equations of second order. Adv. Differ. Equ. 2014, 2014, 294. [CrossRef]

24. Zhao, X.; Zhang, X.; Ge, W. Hyers-Ulam stability of a class second differential equation $y^{\prime \prime}(x)+p(x) y^{\prime}(x)+$ $q(x) y(x)=F(y(x))$. Bull. Malays. Math. Sci. Soc. 2017, 40, 891-906. [CrossRef]

25. Harris, C.J.; Miles, J.F. Stability of Linear Systems: Some Aspects of Kinematic Similarity. Mathematics in Science and Engineering; Academic Press, Inc.: London, UK, 1980.

(C) 2019 by the authors. Licensee MDPI, Basel, Switzerland. This article is an open access article distributed under the terms and conditions of the Creative Commons Attribution (CC BY) license (http:/ / creativecommons.org/licenses/by/4.0/). 